\title{
Case Report \\ Phenotypic and genotypic characterization of Aspergillus lentulus and Aspergillus fumigatus isolates in a patient with probable invasive aspergillosis
}

\author{
Graciela Montenegro, ${ }^{1}$ Silvia Sánchez Puch, ${ }^{1}$ Virginia M. Jewtuchowicz, ${ }^{1}$ \\ Maria V. Pinoni, ${ }^{1}$ Silvia Relloso, ${ }^{1,2}$ Elena Temporitti, ${ }^{2}$ Cristina A. lovannitti ${ }^{1}$ \\ and Maria T. Mujica ${ }^{1}$ \begin{abstract}
Buenos Aires, Paraguay 2150, Piso 11 (1121), Buenos Aires, Argentina
${ }^{2}$ Centro de Educación Médica e Investigaciones Clínicas 'Dr. Norberto Quirno' (CEMIC), Buenos Aires, Argentina
\end{abstract} \\ ${ }^{1}$ Departamento de Microbiología, Parasitología e Inmunología, Facultad de Medicina, Universidad de
}

Correspondence

Maria T. Mujica

mtmujica@gmail.com

Received 16 August 2008

Accepted 21 November 2008
Characteristic morphological and phylogenetic analyses demonstrated the presence of Aspergillus fumigatus and Aspergillus lentulus as the aetiological agents in a case of probable invasive aspergillosis (IA). This is believed to be the first report of an $A$. lentulus strain isolated from a patient with probable IA in Argentina.

\section{Introduction}

Aspergillus fumigatus is the species most commonly associated with invasive aspergillosis (IA). This species affects immunocompromised patients, particularly those who are suffering from severe haematological malignancies and transplant recipients. Aspergillus infections have been reported in 2 $26 \%$ of haematopoietic stem cell transplant recipients and in $1-15 \%$ of organ transplant recipients (Singh \& Paterson, 2005). The IA that follows solid-organ transplantation is most common in heart-lung transplant patients and is found, in decreasing order, in liver, heart, lung and kidney recipients (Singh \& Paterson, 2005; Latgé, 1999). A. fumigatus, the major aetiological agent of IA, is identified by macroscopic and microscopic features (Rapper \& Fennell, 1965) and, despite small variations in phenotype, has been largely regarded as a single species. However, results of molecular studies demonstrate that several phenotypically identified $A$. fumigatus strains might be genetically distinct (Balajee et al., 2005a, b, 2006). Recently, several slowly sporulating $A$. fumigatus isolates have been identified as members of a new species by multilocus sequence typing (Balajee et al., 2005a; Hong et al., 2005). The phylogenetic relationship between $A$. fumigatus and related species has been analysed by sequencing

Abbreviations: AMB, amphotericin B; BAL, bronchoalveolar lavage; CAS, caspofungin; IA, invasive aspergillosis; ITS, internal transcribed spacer; VRZ, voriconazole.

The GenBank/EMBL/DDBJ accession numbers for the $\operatorname{ben} A, \operatorname{rod} A$, mtcytb and ITS sequences of Aspergillus reported in this study are EU254411, EU254412, EU254414 and EU254413 for RS:B2007AR, and EU254415, EU254416, EU254418 and EU254417 for RS:V2007AR. parts of the $\beta$-tubulin-encoding gene (ben $A)$, the mitochondrial cytochrome $b$-encoding gene $(m t c y t b)$, the rodlet Aencoding gene $(\operatorname{rod} A)$, the salt-responsive gene, the internal transcribed spacer (ITS) 1-5.8S-ITS2 region, the genes encoding calmodulin and actin (Balajee et al., 2005b, 2006; Hong et al., 2005; Wang et al., 2000; Yaguchi et al., 2007).

Identification to the species level appears to be important for the genus Aspergillus, since members of this genus may demonstrate variable susceptibility to antifungal drugs (Balajee et al., 2005a, 2006; Dannaoui et al., 2001; Mellado et al., 2006; Walsh et al., 2003). MICs of multiple antifungal agents were higher for atypical isolates than for the typical A. fumigatus belonging to the Aspergillus section Fumigati (Mellado et al., 2006; Balajee et al., 2004).

In this communication, a fatal case of probable IA (De Pauw et al., 2008) in a patient from Argentina is described. Two Aspergillus species closely related to A. fumigatus were simultaneously isolated from three bronchoalveolar lavage (BAL) samples. We also determined the phylogenetic relationship between the isolates, A. fumigatus and related species by multilocus sequence typing, and analysis of the DNA sequences of partial benA, mtcytb and rodA genes, and ITS regions. Furthermore, the relationship between morphological features and susceptibility profiles for voriconazole (VRZ), amphotericin B (AMB) and caspofungin (CAS) of both isolates was analysed.

\section{Case report}

A 43-year-old Argentine woman with arterial hypertension and an end-stage chronic kidney disease of unknown 
aetiology received haemodialysis from 1994 to 2004. She underwent kidney transplantation in May 2005 and her immunosuppressive regimen comprised a combination of mycophenolate mofetil hydrochloride, cyclosporine and prednisolone. However, three graft rejection episodes occurred: the last one was in October 2005.

A month later, she received medical care at Centro de Educación Médica e Investigaciones Clínicas 'Dr. Norberto Quirno' (CEMIC) University Hospital, in the city of Buenos Aires, for fever and respiratory disorders (cough and severe dyspnoea grade III). Chest radiography showed lung infiltrates, suggesting a community-acquired pneumonia related to immunosuppression. She was treated with cefepime and levofloxacine. Blood and sputum cultures were negative for fungi and bacteria.

As her general clinical health deteriorated and new infiltrates were observed by chest radiography, an inotropic therapy was necessary. Fibrebronchoscopy was performed before initiating an empirical antibiotic and antifungal therapy with imipenem, levofloxacine and liposomal AMB (AmBisome). The BAL culture was again negative for fungal and bacterial growth. Eleven days after hospitalization, an unfavourable progression of the symptoms was reported and computed axial tomography (CAT) scans were performed. The CAT scans revealed patchy areas of consolidation and three nodular lesions in lungs. Three new BAL cultures showed microscope evidence of the presence of septate, hyaline and dichotomously branched hyphae, whereas BAL cultures on blood agar yielded abundant growth of 2 Aspergillus species (between 5 to 8 and 10 to 12 colonies of each Aspergillus isolate from 3 BAL samples were simultaneously isolated and identified). In serum, $3.9 \mathrm{ng}$ Aspergillus galactomannan $\mathrm{ml}^{-1}$ was detected by ELISA (Platelia Aspergillus; Bio-Rad) (Bretagne et al., 1997; Stynen et al., 1995). Based upon data from the EORTC/MSG (European Organization for Research and Treatment of Cancer/Mycoses Study Group) (De Pauw et al., 2008), a diagnosis of probable invasive fungal lung infection was made. The patient then received an inotropic therapy with liposomal AMB. A total dose of $5.0 \mathrm{~g}$ was administered over a period of 30 days. The patient finally died of multiple organ failure in January 2006. An autopsy of the corpse had not been performed at the time of writing.

Two fungal strains were isolated from BAL on blood agar culture and evaluated for growth characteristics (radial growth and conidiation) on Czapek-Dox agar (CZD; Becton Dickinson) at $37{ }^{\circ} \mathrm{C}$. These two strains were named: RS : B2007AR and RS:V2007AR, and their growth on CZD was similar. One isolate, RS : B2007AR, grew as fluffy white colonies consisting predominantly of vegetative hyphae after a 7 day incubation. After prolonged incubation (10 days), it resembled the isolate RS:V2007AR, except for reduced sporulation, so that colonies were pale green blue rather than deep blue. Growth of both isolates was evaluated at 48 and $50{ }^{\circ} \mathrm{C}$ on CZD agar plates containing
Aspergillus minimal agar medium (Balajee et al., 2005b). There was a growth difference between the two fungal strains isolated when incubated at high temperature. Isolate RS: B2007AR did not grow at $50{ }^{\circ} \mathrm{C}$, although it grew poorly at $48{ }^{\circ} \mathrm{C}$. However, isolate RS:V2007AR grew at both temperatures.

In vitro tests of susceptibility to antifungal agents performed according to Clinical Laboratory Standards Institute (CLSI) guidelines (CLSI, 2002) for the RS : B2007AR isolate showed that both the MICs for VRZ and $\mathrm{AMB}$, and the minimum effective concentrations (Kurtz et al., 1994) for CAS, were high (VRZ and AMB, $2 \mu \mathrm{g} \mathrm{ml}^{-1}$; CAS, $>16 \mu \mathrm{g} \mathrm{ml}^{-1}$ ). The RS:V2007AR isolate also showed a normal-sporulation phenotype and susceptibility to AMB $\left(0.5 \mu \mathrm{g} \mathrm{ml}^{-1}\right), \operatorname{VRZ}\left(0.5 \mu \mathrm{g} \mathrm{ml}^{-1}\right)$ and CAS $\left(0.125 \mu \mathrm{ml}^{-1}\right)$.

Genes $\operatorname{rodA}(481 \mathrm{bp})$, mtcytb (437 bp) and benA (471 bp), as well as the ITS region (596-598 bp) of the rRNA gene were amplified by PCR following the protocol described by Balajee et al. (2005b) and Wang et al. (2000). Subsequently, the amplicons were sequenced in order to perform phylogenetic studies. Sequences of benA, rodA, mtcytb and ITS region of the Aspergillus isolates obtained in this study have been submitted to GenBank and assigned the following accession numbers: EU254411, EU254412, EU254414 and EU254413 for RS:B2007AR, and EU254415, EU254416, EU254418 and EU254417 for RS : V2007AR.

Nucleotide sequences for ITS, and $\operatorname{rodA}$, benA and mtcytb genes of the RS: V2007AR and RS:B2007AR isolates were aligned with nucleotide sequences of A. fumigatus and other related species deposited in the GenBank using the CLUSTAL W program. Phylogenetic analyses were performed using the neighbour-joining method, while phylogenetic trees were obtained by bootstrapping using 1000 replicates with the MEGA program. The sequence identity matrix and entropy plot were built using the BioEdit program (data not shown). It was observed that the nucleotide sequences of $\operatorname{rodA}$, benA and mtcytb genes of RS:V2007AR and RS: B2007AR isolates clustered together with those of $A$. fumigatus and Aspergillus lentulus, respectively (Figs 1a and $2 \mathrm{a}, \mathrm{b})$. For both strains, the phylogenetic trees obtained with $\operatorname{rod} A$ and $b e n A$ genes showed a bootstrap value higher than 50 , whereas with the $m t c y t b$ gene the bootstrap value exceeded 60 for RS: B2007AR, and 50 for RS:V2007AR. In the phylogenetic tree of the ITS region, the RS:B2007AR strain was not included exactly in either the A. fumigatus group or the A. lentulus groups, unlike the rest of the analysed genes. Analysis of the nucleotide sequence alignment of the ITS region (Fig. 1b) showed that the non-inclusion of the RS:B2007AR strain in the aforesaid groups was due to a change of nucleotides at positions 59 $(\mathrm{C} \rightarrow \mathrm{T})$ and $138 \quad(\mathrm{~A} \rightarrow \mathrm{G})$. This nucleotide substitution detected in the RS:B2007AR strain may suggest that it could be included in the A. fumigatus or A. lentulus group. The numbers of small changes in the nucleotide among 
(a)

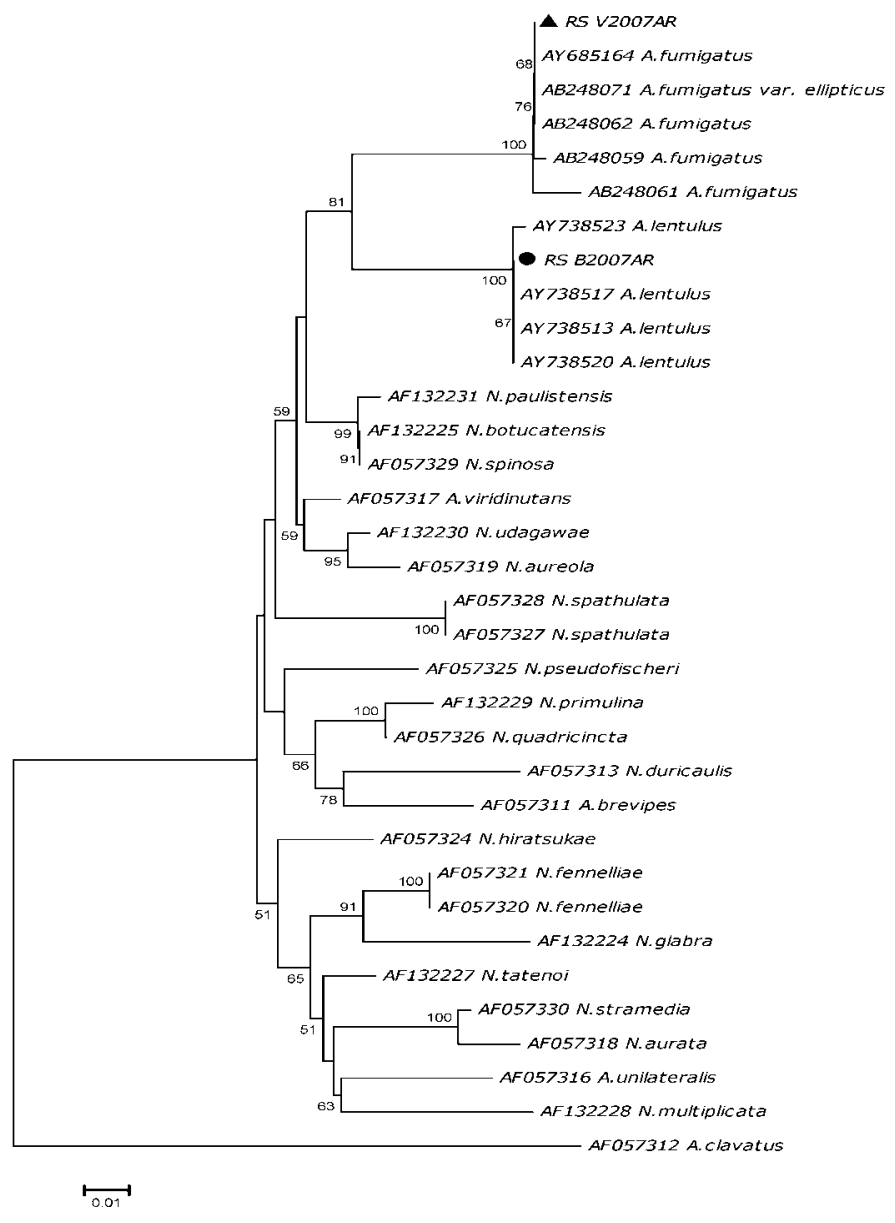

(b)

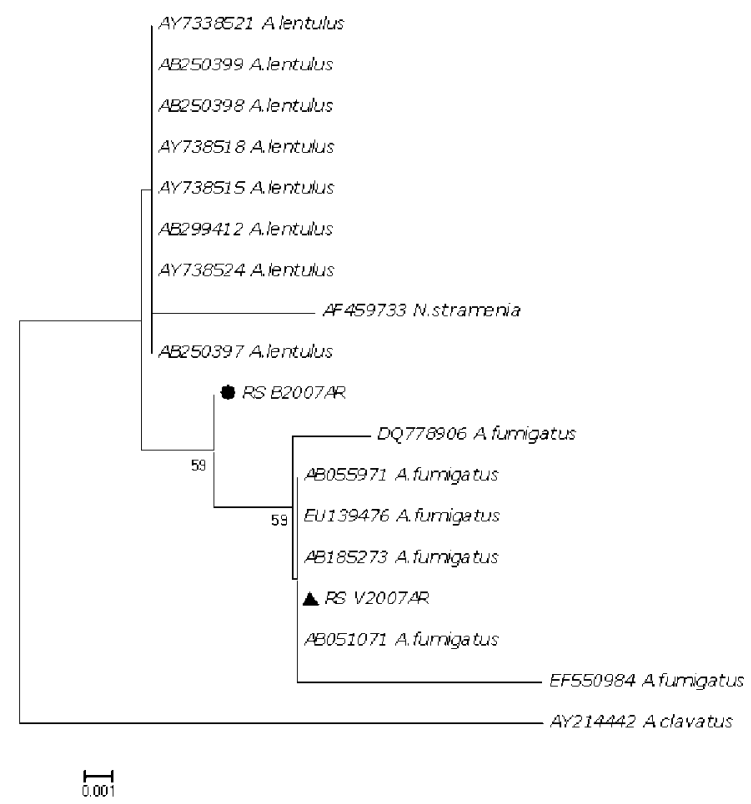

Fig. 1. Phylogenetic tree inferred from neighbour-joining analysis of partial (a) benA gene and (b) ITS region sequences. The sequences derived in this study are denoted by $\boldsymbol{\Lambda}$ and - symbols. Numbers above the nodes represent bootstrapping values. These values were generated from 1000 replicates. The bar length represents the specified genetic distance. RS : V2007AR and RS : B2007AR isolates correspond to A. fumigatus and A. lentulus, respectively. A., Aspergillus; N., Neosartorya.

taxa are represented by the long arms of both $A$. fumigatus and A. lentulus.

The identity percentages of the nucleotide sequences of the strains with respect to GenBank nucleotide sequences were obtained. For the four sequences, the RS: V2007AR strain was observed to have a 98 to $100 \%$ identity with $A$. fumigatus, while the RS:B2007AR strain was observed to have a 96 to $100 \%$ identity with $A$. lentulus. It was observed that $\operatorname{ben} A$ and $\operatorname{rod} A$ genes have higher variability than the $m t c y t b$ gene and the ITS region in their nucleotide sequences. When the entropy of each of the genes and the ITS region were analysed as a measure of variability, it was found that the $\operatorname{ben} A$ and $\operatorname{rod} A$ genes had a mean entropy value of 0.2 each, while the mean values in the $m t c y t b$ gene and the ITS region were $0.02-0.03$, respectively. The maximum entropy value was observed in the ITS region among nucleotide positions 59 and 138 . The noninclusion of the RS : B2007AR strain in the aforesaid groups was due to the high variability of those nucleotide positions. Thus, it was determined that the two strains isolated from the clinical sample belonged to two different species of Aspergillus. One strain, RS:V2007AR, was identified as $A$. fumigatus, and the other one, RS : B2007AR, as A. lentulus.

\section{Discussion}

The increasing frequency of IA and the high mortality associated with disseminated fungal diseases have highlighted the need for a rapid microbiology diagnosis and identification of isolated fungi (Singh \& Paterson, 2005). Our patient presented with two strains of Aspergillus simultaneously. One strain, RS:V2007AR, presented typical characteristics belonging to A. fumigatus, and the other one, RS:B2007AR, was identified as an atypical $A$. fumigatus. They were classified according to phenotypic 
(a)

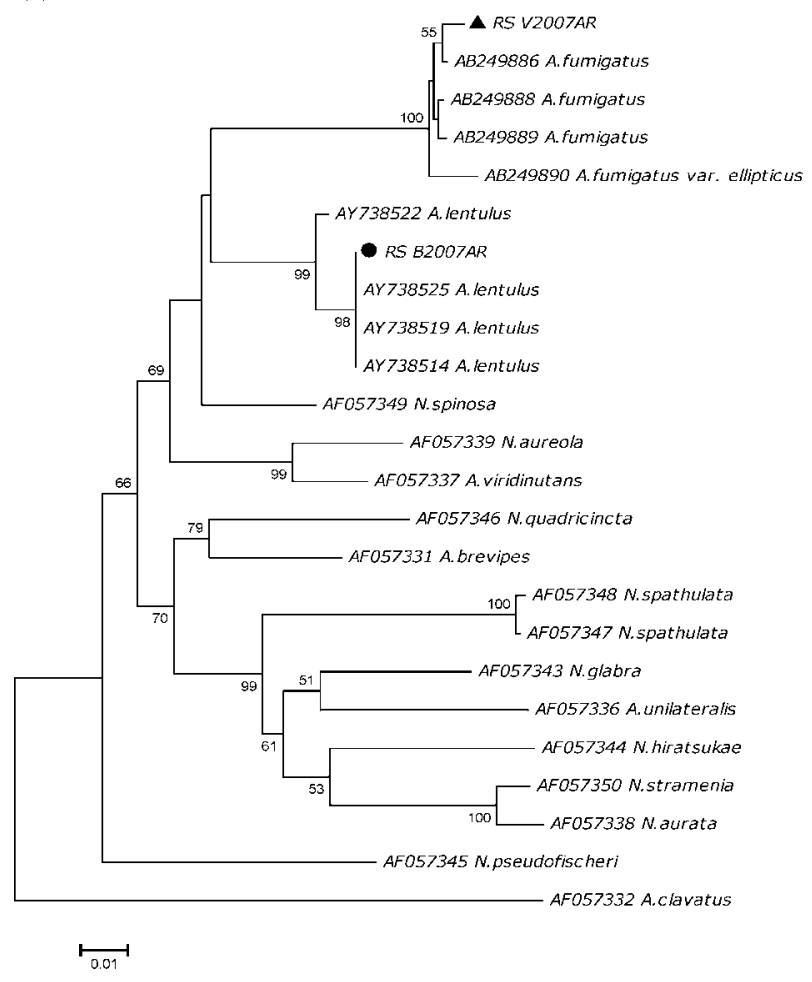

(b)

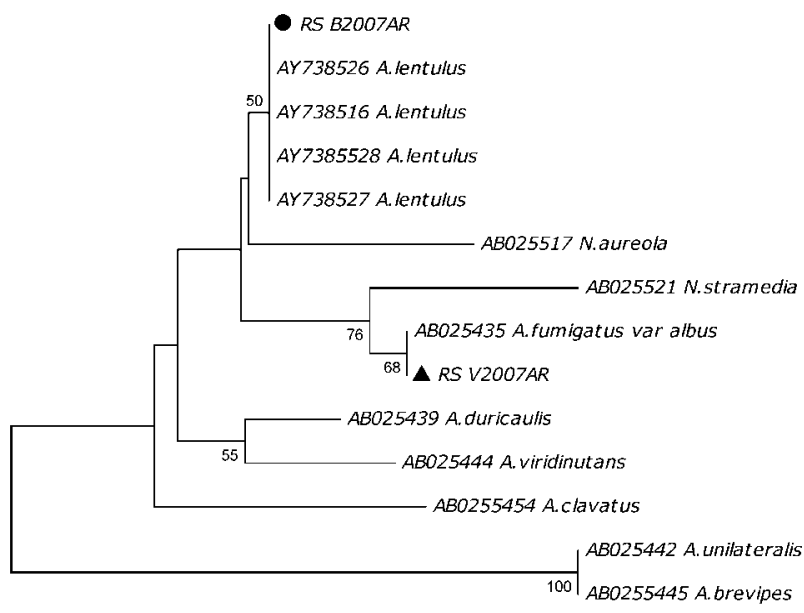

$\stackrel{\mapsto}{\stackrel{1}{1}}$

Fig. 2. Phylogenetic tree inferred from neighbour-joining analysis of partial (a) rodA and (b) mtcytb gene sequences. The sequences derived in this study are denoted by $\boldsymbol{\Delta}$ and $\boldsymbol{0}$ symbols. Numbers above the nodes represent bootstrapping values. These values were generated from 1000 replicates. The bar length represents the specified genetic distance. RS : V2007AR and RS :B2007AR isolates correspond to A. fumigatus and A. lentulus, respectively. A., Aspergillus; N., Neosartorya.

characteristics as A. fumigatus and included in the section Fumigati. Typical A. fumigatus is the predominant agent of IA in an immunocompromised host. However, infections with atypical variants that differ in minor morphological features have been reported (Balajee et al., 2005a, b; Katz et al., 2005; Lass-Flörl et al., 1998). Genetic polymorphisms of A. fumigatus isolates within the same specimens from patients with IA were observed. Patients who carried more than one genotype or concurrent infection by at least two different strains were identified (Bertout et al., 2001; Menotti et al., 2005). The phenotypic characteristics of our RS : B2007AR isolate were observed in A. lentulus (Balajee et al., 2006; Hong et al., 2005). Of note, this isolate had a slow sporulating growth, since it did not grow at $50{ }^{\circ} \mathrm{C}$, although it poorly grew at $48{ }^{\circ} \mathrm{C}$ and was less susceptible to all antifungal drugs tested.

Although both isolates demonstrated different end points to the tested antifungals, the patient's response to therapy was uncertain since treatment failure is generally related to the patient's impaired immunity. Genetic variants of $A$. fumigatus, originally identified as poorly sporulating strains with reduced susceptibility to multiple antifungal agents, have been described. All these atypical Aspergillus strains belonged to the Aspergillus section Fumigati (Balajee et al., 2006; Mellado et al., 2006; Walsh et al., 2003; Balajee et al., 2004; Samson et al., 2006).

Identification and classification of filamentous fungi rely mainly on morphological criteria, but the limitations of phenotypic typing of fungal pathogens are increasingly recognized (Balajee et al., 2005a, 2006). Identification of the most common species in the section Fumigati remains problematic due to the variability of phenotypic characteristics (Rapper \& Fennell, 1965), but no great variation was observed when $A$. fumigatus strains were analysed by $m t c y t b$, ben $A$ and $\operatorname{rod} A$ genes, ITS regions and calmodulinencoding gene sequence analysis (Balajee et al., 2005b; Hong et al., 2005; Wang et al., 2000). In this study, the entropy profiles at the nucleotide level demonstrated a significant increase in $\operatorname{ben} A$ and $\operatorname{rod} A$ genes. This result might reflect a fungal attempt to adapt to changing environmental conditions. We believe that it would be more appropriate to use benA and $\operatorname{rod} A$ genes to differentiate among 'species' of Aspergillus by phylogenetic analysis instead of the $m t c y t b$ gene or the ITS region as it has already been indicated (Alcazar-Fuoli et al., 2008). To the best of our knowledge, this is the first report of 
simultaneous infection with $A$. fumigatus and $A$. lentulus in Argentina, and the first report of an A. lentulus isolate from a patient with probable IA in our country.

\section{Acknowledgements}

This research was supported by grant UBACyT-M426, from the Universidad de Buenos Aires. We are grateful to Verónica Mathet $\mathrm{PhD}$, Miss Maria Luján Cuestas and Mr Sergio Mazzini for their excellent technical assistance.

\section{References}

Alcazar-Fuoli, L., Mellado, E., Alastruey-Izquierdo, A., CuencaEstrella, M. \& Rodriguez-Tudela, J. L. (2008). Aspergillus section Fumigati: antifungal susceptibility patterns and sequence-based identification. Antimicrob Agents Chemother 52, 1244-1251.

Balajee, S. A., Weaver, M., Imhof, A., Gribskov, J. \& Marr, K. A. (2004). Aspergillus fumigatus variant with decreased susceptibility to multiple antifungals. Antimicrob Agents Chemother 48, 1197-1203.

Balajee, S. A., Gribskov, J. L., Brandt, M., Ito, J., Fothergill, A. \& Marr, K. A. (2005a). Mistaken identity: Neosartorya pseudofischeri and its anamorph masquerading as Aspergillus fumigatus. J Clin Microbiol 43, 5996-5999.

Balajee, S. A., Gribskov, J. L., Hanley, E., Nickle, D. \& Marr, K. A. (2005b). Aspergillus lentulus sp. nov., a new sibling species of $A$. fumigatus. Eukaryot Cell 4, 625-632.

Balajee, S. A., Nickle, D., Varga, J. \& Marr, K. A. (2006). Molecular studies reveal frequent misidentification of Aspergillus fumigatus by morphotyping. Eukaryot Cell 5, 1705-1712.

Bertout, S., Renaud, F., Barton, R., Symoens, F., Burnod, J., Piens, M.-A., Lebeau, B., Viviani, M.-A., Chapuis, F. \& other authors (2001). Genetic polymorphism of Aspergillus fumigatus in clinical samples from patients with invasive aspergillosis: investigation using multiple typing methods. J Clin Microbiol 39, 1731-1737.

Bretagne, S., Marmorat-Khuong, A., Kuentz, M., Latgé, J.-P., BartDelabesse, E. \& Cordonnieret, C. (1997). Serum Aspergillus galactomannan antigen testing by sandwich ELISA: practical use in neutropenic patients. J Infect 35, 7-15.

CLSI (2002). Reference Method for Broth Dilution Antifungal Susceptibility Testing of Filamentous Fungi, approved standard M38A. Wayne, PA: Clinical Laboratory Standards Institute.

Dannaoui, E., Borel, E., France-Monier, M., Piens, M. A., Picot, S. \& Persat, F. (2001). Acquired itraconazole resistance in Aspergillus fumigatus. J Antimicrob Chemother 47, 333-340.

De Pauw, B., Walsh, T. J., Donnolly, J. P., Stevens, D. A., Edwards, J. E., Calandra, T., Pappas, P. G., Maertens, J., Lortholary, O. \& other authors (2008). Revised definitions of invasive fungal disease from the European Organization for Research and Treatment of Cancer/ Invasive Fungal Infections Cooperative Group and the National
Institute of Allergy and Infectious Disease Mycoses Study Group (EORTC/MSG) consensus group. Clin Infect Dis 46, 1813-1821.

Hong, S. B., Go, S. J., Shin, H. D., Frisvad, J. C. \& Samson, R. A. (2005). Polyphasic taxonomy of Aspergillus fumigatus and related species. Mycologia 97, 1316-1329.

Katz, M. E., Dougall, A., Weeks, K. \& Cheetham, B. (2005). Multiple genetically distinct groups revealed among clinical isolates identified as atypical Aspergillus fumigatus. J Clin Microbiol 43, 551-555.

Kurtz, M. B., Heath, L. B., Marrinan, J., Dreikorn, S., Onishi, J. \& Douglas, C. (1994). Morphological effects of lipopeptides against Aspergillus fumigatus correlate with activities against (1,3)- $\beta$-D-glucan synthase. Antimicrob Agents Chemother 38, 1480-1489.

Lass-Flörl, C., Kofler, G., Kropshofer, G., Hermans, J., Kreczy, A., Dierich, M. P. \& Niederwieser, D. (1998). In-vitro testing of susceptibility to amphotericin B is reliable predictor of clinical outcome in invasive aspergillosis. J Antimicrob Chemother 42, 497502.

Latgé, J. P. (1999). Aspergillus fumigatus and aspergillosis. Clin Microbiol Rev 12, 310-350.

Mellado, E., Alcazar-Fuoli, L., García-Effrón, G., Alastruey-Izquierdo, A., Cuenca-Estrella, M. \& Rodríguez-Tudela, J. L. (2006). New resistance mechanisms to azole drugs in Aspergillus fumigatus and emergence of antifungal drugs-resistant A. fumigatus atypical strains. Med Mycol 44, S367-S371.

Menotti, J., Waller, J., Meunier, O., Letscher-Bru, V., Herbrecht, R. \& Candolfi, E. (2005). Epidemiological study of invasive pulmonary aspergillosis in a haematology unit by molecular typing of environmental and patient isolates of Aspergillus fumigatus. J Hosp Infect 60, 61-68.

Rapper, K. B. \& Fennell, D. I. (1965). The Genus Aspergillus. Baltimore, MD: Williams \& Wilkins.

Samson, R. A., Hong, S.-B. \& Frisvad, J. C. (2006). Old and new concepts of species differentiation in Aspergillus. Med Mycol 44, S133S148.

Singh, N. \& Paterson, D. L. (2005). Aspergillus infections in transplant recipients. Clin Microbiol Rev 18, 44-69.

Stynen, D., Goris, A., Sarfati, J. \& Latgé, J. P. (1995). A new sensitive sandwich enzyme-linked inmunosorbent assay to detect galactofuran in patients with invasive aspergillosis. J Clin Microbiol 33, 497-500.

Walsh, T. J., Petraitis, V., Petraitiene, R., Field-Ridley, A., Sutton, D., Ghannoum, M., Sein, T., Schaufele, R., Peter, J. \& other authors (2003). Experimental pulmonary aspergillosis due to Aspergillus terreus: pathogenesis and treatment of an emerging pathogen resistant to amphotericin B. J Infect Dis 188, 305-309.

Wang, L. I., Yokoyama, K., Miyaji, M. \& Nishimura, K. (2000). Mitochondrial cytochrome $b$ gene analysis of Aspegillus fumigatus and related species. J Clin Microbiol 38, 1352-1358.

Yaguchi, T., Horie, Y., Tanaka, R., Matsuzawa, T., Ito, J. \& Nishimura, K. (2007). Molecular phylogenetics of multiple genes on Aspergillus section Fumigati isolated from clinical specimens in Japan. Nippon Ishinkin Gakkai Zasshi 48, 37-46. 